\title{
Establecimiento de un Régimen de Facilitación del Comercio en Chile: Estudio del Comercio Internacional desde la Perspectiva de las Transiciones Socio-técnicas
}

\author{
Miguel A. Muñoz \\ Facultad de Administración y Economía, Universidad Tecnológica Metropolitana, Dr. Hernán Alessandri 722, Santiago, \\ Chile. (e-mail: miguel.munoz@utem.cl)
}

Recibido Mar. 18, 2019; Aceptado May. 9, 2019; Versión final Jun. 19, 2019, Publicado Dic. 2019

\begin{abstract}
Resumen
El objetivo del estudio consiste en analizar la implementación de tecnologías para la facilitación del comercio exterior chileno, utilizando la perspectiva del análisis multinivel de las transiciones socio-técnicas, con un alcance exploratorio. Se realiza un análisis documental de publicaciones periódicas, principalmente revistas chilenas del ámbito, para identificar la relación del nivel macro y de la política comercial con el establecimiento del régimen de facilitación del comercio, entre los años 1961 y 2011. Los resultados muestran que la liberalización del comercio exterior y las instituciones internacionales promotoras del libre comercio han presionado en la implementación de medidas de facilitación del comercio en Chile, ayudando a estructurar el nuevo régimen. Se concluye que el uso de la perspectiva de las transiciones permite evidenciar las presiones del paisaje socio-técnico, así como de la política comercial, en el establecimiento de un régimen de facilitación del comercio basado en tecnologías de la información.
\end{abstract}

Palabras clave: transiciones socio-técnicas; facilitación del comercio; comercio internacional; política comercial

\section{Establishment of a Trade Facilitation Regime in Chile: A Study of International Trade from the Perspective of Sociotechnical Transitions}

\begin{abstract}
The objective of the study is to analyze the implementation of technologies for the facilitation of Chilean foreign trade, using the perspective of multi-level analysis of socio-technical transitions, with an exploratory scope. A documentary analysis of periodical publications is carried out, mainly Chilean journals in the field, to identify the relationship of the macro level and commercial policy with the establishment of the trade facilitation regime, between 1961 and 2011. The results show that the liberalization of foreign trade and international institutions that promote free trade have put pressure on the implementation of trade facilitation measures in Chile, helping to structure the new regime. It is concluded that the use of the perspective of transitions makes it possible to demonstrate the pressures of socio-technical landscape and commercial policy in the establishment of a trade facilitation regime based on information technologies.
\end{abstract}

Keywords: sociotechnical transitions; trade facilitation; international trade; commercial policy 


\section{INTRODUCCIÓN}

Desde la segunda mitad del siglo XX, el comercio internacional ha atravesado por un sostenido proceso de liberalización comercial, sostenido en la construcción de un sistema multilateral encabezado por el Acuerdo General sobre Aranceles Aduaneros y Comercio (GATT, por siglas en inglés), de 1947, y la Organización Mundial de Comercio (OMC), a partir de 1995, junto a otras instituciones internacionales, como el Fondo Monetario Internacional y el Banco Mundial. Un aspecto de ese fenómeno, que ha comenzado a indagarse incipientemente, se refiere a la agilización de los trámites de ingreso y salida de mercancías en los distintos países, o "Facilitación del comercio", el cual es un elemento relevante, por cuanto un proceso de liberalización comercial, que busca incrementar el comercio de bienes, requiere de modo indispensable la gestión ágil y expedita de la información respectiva, a través de las fronteras nacionales. Especialmente, porque dicha información tiene origen en distintos organismos, estatales y privados, que participan en el comercio exterior mediante distintas autorizaciones. Las medidas que buscan facilitar el comercio son complementarias a la liberalización comercial (Zaki, 2015). Su estudio, sin embargo, se ha enfrentado, principalmente, desde la mirada económica. Esta investigación ofrece una perspectiva distinta desde la cual abordar este aspecto, pues se aboca a los cambios socio-técnicos asociados al desarrollo del comercio exterior chileno.

En los últimos años, la "Facilitación del comercio" se ha instituido en un ámbito de interés empujado, especialmente, por los argumentos de competitividad nacional mediante la eliminación del papeleo y de trámites burocráticos (Grainger, 2011). En una nota educativa de su página en la red, la OMC (1998) definió la "Facilitación del comercio" como "la simplificación y armonización de los procedimientos de comercio internacional" entendiendo por tales "las actividades, prácticas y formalidades involucradas en el cobro de impuestos, presentación, comunicación y procesamiento de los datos requeridos para el movimiento de los bienes en el comercio internacional" (Grainger, 2011). Su importancia se ha incrementado, especialmente, desde la suscripción del Acuerdo sobre Facilitación del Comercio, en el marco de la OMC, el cual promueve el desarrollo de distintas capacidades, entre los países miembros, con el propósito de reducir trabas burocráticas (WT/L/940, 2014). Pero son distintos los elementos que coparticipan facilitando el comercio; uno de ellos es la agilización de los despachos aduaneros por medio del uso de tecnologías de la información, por ejemplo, a través de la creación de "ventanillas únicas de comercio exterior" (VUCE), como la que se ha intentado implementar en Chile a lo largo de la última década.

La literatura sostiene que las medidas de facilitación del comercio conllevan beneficios para los distintos países, particularmente, en cuanto a reducciones de tiempos y de costos -los cuales serían bastante menores que los beneficios generados con la implementación de las medidas (Moïsé, 2013)-. También ayudarían a aumentar el volumen de comercio (Moïsé y Sorescu, 2013). Estos beneficios estarían ligados a la mayor disponibilidad de información, la simplificación y armonización de documentos, la racionalización de los procedimientos y el uso de procedimientos automatizados (Moïsé y Sorescu, 2013). Por otro lado, Dennis y Shepherd (2011) sostienen que la facilitación puede promover incluso la diversificación de exportaciones en los países en vías de desarrollo. Una de las principales herramientas de facilitación del comercio se refiere a las tecnologías de la información y la comunicación (TIC): de hecho, las investigaciones muestran casos exitosos, como los de la República de Corea (Yang, 2010), y otros, como India y Sri Lanka, en los cuales las tecnologías informáticas dirigidas a la facilitación del comercio se han implementado en conjunto con una política de apertura comercial (Rana, 2012; Wijayasiri y Jayaratne, 2010). No obstante, Moradhasel et al. (2008) afirman que la implementación de TIC en ese ámbito ejerce un efecto positivo evidenciable en países desarrollados, pero no en países en vías de desarrollo, probablemente porque hay pruebas de correlación positiva entre el dominio de esas tecnologías y la facilitación del comercio, a través del mejoramiento del ambiente de negocios, y de la infraestructura de transporte y comunicaciones, lo cual no se evidenciaría en países en vías de desarrollo (Katos, 2010). Y, más específicamente, se ha afirmado que una modernización tecnológica, como la ejecución de programas de VUCE, mejoran el desempeño comercial de los países (De Sá Porto y Morini, 2014).

En suma, el aspecto tecnológico cumple un papel importante en el comercio internacional y constituye una dimensión que ha sido abordada por distintos autores en la última década, desde perspectivas económicas cuantitativas. Sin embargo, este trabajo ofrece un punto de mirada diverso sobre esta cuestión, pues se centra en los cambios socio-técnicos aparejados al desarrollo del comercio exterior. Es decir, se apoya en el ámbito de los estudios sociales de la tecnología, puesto que no se enfoca en aparatos técnicos considerados aisladamente, sino en "la red sin costura" de la que tales artefactos son apenas una parte entramada con actores sociales, políticas, valores y con otros artefactos (Hughes, 1983). Igualmente, es tributario de las contribuciones hechas desde la perspectiva de la economía evolutiva sobre el cambio tecnológico, las cuales han abordado el estudio de la innovación desde un enfoque sistémico. Sin embargo, estos desarrollos no dan cuenta de las trayectorias que experimentan las relaciones socio-técnicas, ni los distintos niveles del entramado social, cuestiones que sí considera, en cambio, la perspectiva del análisis multi-nivel de las transiciones socio-técnicas (Geels, 2002). 
Las transiciones socio-técnicas consisten en "el cambio desde una configuración socio-técnica a otra, incluyendo sustitución de tecnología, así como cambios en otros elementos" (Geels, 2002), todos los cuales "no existen autónomamente, sino que son creados, reproducidos y refinados por grupos sociales" (Geels, 2005a). Las distintas configuraciones socio-técnicas corresponden al entramado mediante el cual se satisfacen requerimientos sociales, como, por ejemplo, la provisión de energía eléctrica o de agua potable. De este modo, la transición supone un cambio paulatino desde un sistema o configuración socio-técnica a otra, en la cual participan innovaciones tecnológicas, "por ejemplo, las transiciones desde un sistema de transporte basado en carruajes empujados por caballos a un sistema de transporte basado en automóviles" o desde un sistema de saneamiento basado en pozos negros hacia sistemas integrados de alcantarillado (Geels 2005b; Geels y Kemp, 2007).

Las transiciones socio-técnicas pueden abordarse a partir del análisis de la interacción de diferentes niveles sociales: micro, meso y macro (Geels, 2012). El nivel micro, también llamado nicho socio-técnico, corresponde al espacio en el cual aparecen las innovaciones tecnológicas radicales, se incuban y son protegidas de la competitividad del mercado mientras las condiciones no le permitan competir. Este nivel está constituido por una red de actores inestable, reglas "vagas e imprecisas" y distintas visiones de los actores acerca del producto. Una vez que las redes sociales en torno a la nueva tecnología se amplíen y las reglas que coordinan su acción se hayan vuelto más estables, el nicho se habrá proyectado en otro nivel social, un régimen sociotécnico, o "nivel meso" de análisis, caracterizado por una mayor estructuración. La coevolución de distintas trayectorias (ciencia, tecnología, infraestructuras, políticas, industrias, mercados y comportamiento de usuarios, por ejemplo) alcanza en este nivel una estabilidad dinámica, es decir, una "estabilidad que no es inercia...lo que significa que la innovación aún ocurre, pero de modo incremental" (Geels, 2005a). El régimen consiste, entonces, en un conjunto semi-coherente de reglas, que conforman un contexto coordinado que orienta la acción. Las reglas son reforzadas y (re)producidas por los actores, proveyendo estabilidad al régimen a través de mecanismos de cierre, como creencias compartidas, "que hacen a los actores ciegos ante desarrollos fuera de su rango de visión", leyes, normas y otras regulaciones, por mencionar algunos (Geels, 2012). Por último, el paisaje socio-técnico, relativo al nivel macro, se refiere al amplio ambiente que afecta al desarrollo socio-técnico y que está más allá de la influencia de los actores.

La transición socio-técnica se produce en tanto los tres niveles mencionados interactúan en una dinámica en que "los desarrollos del paisaje socio-técnico crean presiones sobre el régimen, produciendo problemas...Los actores del régimen reaccionan con ajustes en el sistema, pero no son capaces de resolverlos. Esto crea una ventana de oportunidad para nuevas innovaciones, desarrolladas en los nichos y empujada por una nueva red de grupos sociales" (Geels y Kemp, 2007). Como es evidente, se trata de una perspectiva que no identifica la existencia de un elemento causal, sino de procesos en múltiples dimensiones y niveles, que se refuerzan de forma mutua, por lo que es posible, por ejemplo, que las innovaciones aparecidas en los nichos no prosperen proyectándose como régimen (Geels, 2012). En definitiva, esta investigación tiene por propósito ofrecer una reflexión sobre la dimensión socio-técnica del comercio internacional, desde la perspectiva de las transiciones. Esto se realizará por medio del análisis, a nivel exploratorio, de la trayectoria chilena de implementación de tecnologías para la "facilitación del comercio", centrándose en las presiones ejercidas por el "paisaje socio-técnico" y la política comercial chilena en el establecimiento del "régimen de facilitación del comercio". El período comprendido es entre 1961, cuando arribó a Chile el primer computador utilizado en el desarrollo de tareas aduaneras, y 2011, cuando se llevaron a cabo los esfuerzos de implementación de la VUCE llamada Sistema Integrado de Comercio Exterior (SICEX). Un aspecto relevante de esta indagación radica, precisamente, en que el estudio del comercio exterior se ofrece desde una perspectiva novedosa, la cual, en lugar de enfatizar en su dimensión estrictamente económico-cuantitativa y de estrategias de negocio, se enfoca en los cambios socio-técnicos que le han acompañado. Y, especialmente, en un caso, como el chileno, cuyo contexto contemporáneo se caracteriza por presentar una arraigada política de liberalización comercial, que se ha manifestado en un notable incremento de las exportaciones e importaciones de bienes, desde el año 2003, como puede apreciarse en la Figura 1.

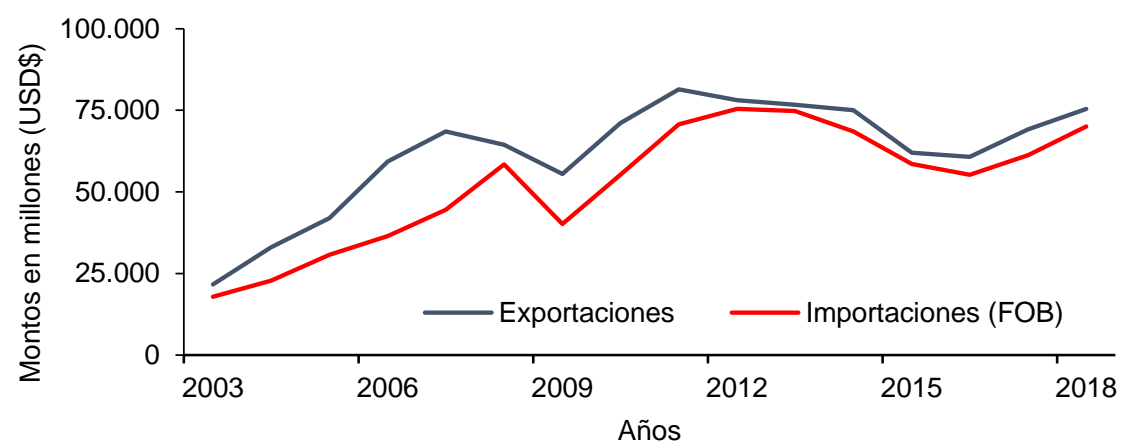

Fig. 1: Montos de Exportaciones e Importaciones chilenas v/s Años (Datos tomados de Banco Central de Chile, 2019) 


\section{METODOLOGÍA}

Se realizó un estudio cualitativo, pues no se sirve de mediciones numéricas de datos, sino de análisis de fuentes documentales, particularmente, de publicaciones periódicas, como boletines, revistas o prensa. Además, se recurre a un enfoque no utilizado hasta ahora sobre el comercio internacional: el de las transiciones socio-técnicas, mediante un análisis multi-nivel. A raíz de esto, se acerca a su objeto de estudio de modo exploratorio (Hernández et al., 2010). Las fuentes analizadas son, especialmente, publicaciones periódicas centradas en la divulgación de información relativa al comercio exterior chileno en el período comprendido entre 1961 y 2011: principalmente, las revistas "Aduana" y "Chile Portuario". El tratamiento dado a estas fuentes ha consistido en un análisis documental de contenido (Hernández et al., 2010), siguiendo un proceso en "espiral" (Creswell, 2007): los datos históricos son recogidos y estudiados para organizarlos en categorías; estas evidencias reunidas se analizan para construir una interpretación de conjunto (a la luz de la literatura sobre la cuestión), que se enriquece con nuevas revisiones de las fuentes hasta su saturación: cuando los datos acopiados ya no ofrecen información novedosa (Hernández et al., 2010). Así, pues, las etapas de acopio y análisis de los datos se interrelacionan y se desarrollan simultáneamente. Los cierres temporales se explican por la llegada a Chile del primer computador a usarse en el procesamiento aduanero, en el año 1961, y por el impulso, en 201, a la ventanilla única denominada "Sistema integrado de Comercio Exterior" (SICEX).

\section{RESULTADOS Y DISCUSIÓN}

En este apartado se exponen los resultados de la indagación, ordenados en función de la trayectoria que ha experimentado la agilización de los procesos operativos del comercio exterior, conformando un "régimen de facilitación del comercio", de acuerdo a la perspectiva de las transiciones socio-técnicas analizadas en multinivel.

\section{Aparición de tecnologías a nivel de nicho}

Las tareas asociadas con la facilitación del comercio no han permanecido ajenas a los cambios tecnológicos que han ocurrido a lo largo del tiempo. El Servicio Nacional de Aduanas de Chile, una institución esencial en el control de las operaciones de comercio exterior, es ejemplo de ello. Así lo deja claro la llegada de su primera computadora, en el año 1961. Se trataba del modelo IBM1401, arribado al Departamento de estadísticas del Servicio. Su recepción fue con gran entusiasmo, si bien su uso se limitó a la operación de trámites estadísticos propios del Servicio, y no abarcó el desarrollo de las tramitaciones aduaneras de importación o exportación. Con su llegada, entonces se afirmaba que el Servicio se estaba poniendo en la vanguardia del desarrollo de sistemas mecanizados en el país. Para concretar su funcionamiento, la otrora Superintendencia de Aduanas suscribió un contrato con la compañía IBM, el cual le permitiría mecanizar algunos de sus procedimientos, beneficiando, principalmente, a importadores, agentes de aduana, funcionarios aduaneros y, por cierto, las distintas aduanas del país. Para ello, operarios del Servicio debieron concurrir a la Ciudad de Buenos Aires a recibir la capacitación pertinente ("El funcionamiento del computador electrónico 1401 es un gran paso para la estadística", 1962).

Las agencias de aduana, al igual que hoy, se encargaban de gestionar la tramitación aduanera, vinculando al Servicio de Aduanas con los actores del comercio exterior. En este período comenzaron a asumir un rol preponderante, principalmente, en virtud de su asociación mediante la conformación de la "Cámara Aduanera", una entidad gremial fundada en 1939, que entonces participaba permanentemente en las discusiones públicas en torno al desarrollo del comercio exterior chileno y las políticas comerciales sostenidas en el país, orientadas por la Industrialización por Sustitución de Importaciones (ISI) (Mewes, 1980)

Para la llegada del computador IBM 1401 al Servicio Nacional de Aduana fue determinante la integración del país en organizaciones como la Asociación Latinoamericana de Libre Comercio, así como la implantación de la Nomenclatura Arancelaria de Bruselas, entre otros acuerdos internacionales, debido a que implicaban el compromiso de llevar a cabo esfuerzos por agilizar los trámites de la operación comercial de ingreso y salida de mercancías. Igualmente, el Programa de Asistencia Técnica acordado entre el Ministerio de Hacienda de Chile y la Agencia para el Desarrollo Internacional, representada por la Misión Económica Estadounidense de 1964, encabezada por Robert Kennedy, contempló entre sus propuestas la agilización de las tareas aduaneras, lo cual se vio reflejado en la implementación del "Departamento Automatizado de Operaciones y Estadística" del Servicio chileno (Kennedy, 1964). En este período, además, el sistema multilateral de comercio comenzaba a organizarse por medio de normas e instituciones, como el GATT, convenio suscrito en 1947, que fue creciendo en integrantes hasta 1994, cuando se estableció la OMC sobre la base del mismo GATT. La relevancia del GATT en este aspecto radica en que en su articulado hace referencia a la necesidad de reducir y simplificar las formalidades y exigencias relativas a documentación, en las operaciones de comercio exterior. También parecen tener un rol preponderante organismos como la Organización Mundial 
de Aduanas (OMA). Ésta, surgida en 1952 como "Consejo de Cooperación Aduanera", se ha empeñado, desde su fundación, en propagar medidas que contribuyan a facilitar las operaciones aduaneras propias del comercio internacional. Los acuerdos más destacables, en este marco, son la Convención Internacional sobre Simplificación y Armonización de Procedimientos Aduaneros (Convención de Kioto), de 1974 y la Convención Revisada de Kioto, de 1999 (Grainger, 2011).

\section{Alineamiento de distintas trayectorias}

En el comercio exterior participa una variedad de actores. $Y$ ha sido así al menos durante todo el período cubierto, si bien han experimentado mutaciones y se han incorporado otros nuevos. No obstante, en cuanto a innovaciones relacionadas con los trámites de despacho de ingreso y de salida de mercancías, parecen ser esenciales la nueva perspectiva económica establecida por el gobierno militar y, como parte de aquella, la integración de Chile en el comercio internacional, es decir, su política de apertura comercial con distintas naciones y con organismos multilaterales. Esa estrategia, si bien fue iniciada en 1974 (mediante un tipo de apertura unilateral, que suponía compromisos adquiridos por Chile y no por la contraparte), se comenzó a profundizar durante la década de 1990, por medio de una estrategia bilateral y de apertura regional, que implicó la suscripción de acuerdos de complementación económica, principalmente con países latinoamericanos. Posteriormente, con el nuevo siglo, la estrategia comercial se amplió más aún con las negociaciones y firmas de Acuerdos de Libre Comercio (Dirección General de Relaciones Económicas Internacionales, 2009). Así pues, la influencia de la política comercial parece fundamental, como podrá notarse.

Juntamente con la incorporación de computadoras al Servicio Nacional de Aduanas, la revisión tanto de algunos boletines del mencionado organismo, como de las Revistas de funcionarios de Aduana y Portuarios, dan cuenta de la evolución institucional que experimentó la tramitación de exportaciones e importaciones. Desde luego, esta situación debe ser contextualizada. Buena parte de las modificaciones aplicadas a esos procedimientos se comenzaron a idear e implementar con el inicio de la dictadura militar. Es posible evidenciar que el concepto "racionalización" comenzó a adquirir un protagonismo significativo en los cambios que fueron llevándose a cabo desde 1974, en lo relativo a procedimientos, todos los cuales obedecen, desde luego, a la política de apertura comercial que se estableció justamente a partir de ese año, mediante drásticas reducciones arancelarias unilaterales (Dirección General de Relaciones Económicas Internacionales, 2009). Es decir: no podía asumirse el riesgo de una política comercial abierta, que incentivara el ingreso de importaciones, así como también los envíos al exterior (estimulados con de la creación de Prochile, por ejemplo, en 1974), eludiendo el peso que significaban las exigencias documentales en la frontera y la necesaria agilización de los procedimientos. Así, por ejemplo, el presidente de la Cámara Aduanera de Chile, señalaba, en 1977, que el Decreto de Ley n743, de 1974 (que modificó la Ordenanza de Aduanas), había impactado en la agilización de los despachos aduaneros en Chile y también había servido de inspiración para las aduanas del resto de los países sudamericanos (Tellería, 1977). Dicho Decreto instituía, fundamentalmente: la erección del "Principio de Buena Fe", como una forma de institucionalidad básica en la relación con los agentes de aduana; la reducción de la duplicidad de funciones que existía entre el representante del Servicio de Aduana y el Agente de Aduanas, lo cual empuja a una reducción de costos; y, por último, el Decreto institucionalizó también el principio de subsidiaridad, de acuerdo con el cual el estado delegó funciones en actores privados capaces de desempeñar las mismas tareas, pero con mayor especialidad, gracias a su experticia (Tellería, 1977).

Bajo la misma lógica, en 1979, el gobierno, animado por el propósito de lograr una gestión pública moderna y racionalizada, así como de reducir la lentitud de trámites de despacho de mercancías, a saber, simplificando la gestión de operaciones aduaneras, estableció un "Reglamento de Operaciones Aduaneras", difundido en el medio como "ROA", con el cual se establecieron cambios en los procedimientos. Por ejemplo: la formalización de las operaciones de ingreso y salida de mercancías dejaron de ejecutarse a través de "pólizas" y pasaron a denominarse "declaraciones", las cuales habrían de ser presentadas físicamente ante el Servicio Nacional de Aduanas por medio de los despachadores de aduana. Estas modificaciones supusieron mayores obligaciones para los usuarios de los servicios aduaneros, puesto que ahora debieron comenzar a preocuparse, más que antes, por que la información que entregaban al despachador de aduana fuera exacta, pues, de lo contrario, el afectado sería el mismo interesado, que vería retrasados sus envíos o importaciones (Fuentes, 1979). A partir del año 1983 comenzó a implementarse una nueva herramienta tecnológica: el Sistema de Comercio Exterior Integral (SICOMEXIN), desarrollado por el Servicio Nacional de Aduanas, el cual utilizó la capacidad de las computadoras en la tramitación de tareas aduaneras, particularmente a través de su subsistema para el procesamiento de declaraciones de importación y de exportación. Entre los beneficios que ofrecía la incorporación del sistema se cuenta la posibilidad de realizar el cálculo directo, en el sistema computacional, de los derechos aduaneros y otros gravámenes generados por una operación de importación, así como el mayor control sobre los formularios de operaciones aduaneras (Llamazales, 1985). 
A pesar de las varias críticas que recibió el Servicio Nacional de Aduanas, en orden a superar los problemas burocráticos que todavía le afectaban (Allard, 2004), un nuevo cambio relevante vino a darse recién al entrar el nuevo siglo: en un proyecto de facilitación de los trámites de ingreso y de salida de mercancías, se determinó el establecimiento de solamente dos documentos para la tramitación aduanera de cada una de esas operaciones, los cuales debían gestionarse por medio de la labor de las agencias de aduana: se trataba del Documento Único de Salida (DUS) y de la Declaración de Ingreso (DIN). Sumado a lo anterior, aproximadamente desde mediados de la década de 1990, se inició la implementación del Intercambio Electrónico de Datos (EDI, por sus iniciales en inglés) con el uso de un estándar internacional de la Organización de Naciones Unidas. El Servicio Nacional de Aduanas orientó su uso a la presentación electrónica de los documentos de destinaciones aduaneras (DUS y DIN), atendiendo los requerimientos de distintos actores del comercio exterior, pero especialmente de exportadores, importadores y agencias de aduana. En consecuencia, los documentos DUS y DIN comenzaron a ser elaborados en línea, por el despachador, y enviados mediante una red de valor agregado o VAN (por sus siglas en inglés) al Servicio Nacional de Aduanas. Ya para el año 2003, casi la totalidad de las declaraciones de importación y exportación se ejecutaban electrónicamente, reduciendo el papeleo, los costos y los tiempos de tramitación (Allard, 2004).

En suma, junto con los actores internacionales, la política de apertura comercial iniciada en 1974 hizo necesario el establecimiento de medidas de facilitación y agilización de los trámites aduaneros de importación y exportación de mercancías, con el apoyo esencial de nuevas tecnologías, pero también, mediante la implementación de nuevos procedimientos aduaneros y el otorgamiento de más responsabilidades a los actores privados, particularmente, a las agencias de aduanas. De esta manera, es posible notar cómo los aspectos del paisaje socio-técnico y de la política comercial liberal empujaron la implementación de cambios en la utilización de tecnologías necesarias para la facilitación del comercio, por medio de la reducción de exigencias documentales. Los cambios que se producían, junto a las normas instituidas, fueron dotando, paulatinamente, de mayor coherencia y estructuración la constitución de un régimen socio-técnico de facilitación del comercio.

\section{Régimen socio-técnico de facilitación de comercio}

Desde la década de 2000, el despliegue del régimen de facilitación del comercio se ha ido asentado hasta su estabilización. Sobre todo, en el marco de un proceso de profundización de la apertura comercial bilateral, expresado en la suscripción de acuerdos comerciales con países y bloques industrializados (Canadá, Estados Unidos, Corea del Sur y Unión Europea, por ejemplo) y con grandes economías nacionales (acuerdos comerciales con China e India, por ejemplo). Sumado a ello, durante esta etapa la OMC suscribió, en 2013, uno de sus acuerdos más importantes: precisamente el Acuerdo sobre Facilitación del Comercio, conseguido durante la Conferencia Ministerial de Bali, el cual tiene por propósito agilizar "el movimiento, levante y despacho de las mercancías", facilitar la disponibilidad de información sobre las normas de los miembros de la OMC, armonizarlas e incrementar la automatización mediante la implementación de medios electrónicos (WT/L/940, 2014). Este acuerdo fue ratificado por Chile en el año 2016 y, al año siguiente, entró en vigor para todos los miembros de la OMC. Otros organismos, como el Foro de Cooperación Económica Asia Pacífico (APEC, por sus iniciales en inglés), del cual Chile forma parte, también han participado en la promoción de la facilitación del comercio, mediante acuerdos que han precedido, incluso, al suscrito en la OMC, como dos "Planes de Acción para la Facilitación del Comercio", adoptados en 2002 y en 2007 (Grainger, 2011).

En la misma línea, en Chile prosiguieron especialmente los esfuerzos por reducir el papeleo mediante el proyecto de Integración de Sistemas por Internet para el Desarrollo de las Operaciones y Regulaciones Aduaneras (ISIDORA), a partir del año 2002, el cual incluyó, entre sus productos, el establecimiento de un sistema electrónico de manifiestos marítimos, terrestres y aéreos, en un sistema de ingreso y otro de salida de información (Allard, 2004). De modo paralelo, en 2003 comenzó a concebirse el desarrollo de una VUCE -como un nuevo empeño en el gobierno de tecnologías de la información (Valencia et al., 2018)-, que permitiera a los usuarios realizar sus trámites de visados y certificaciones para operaciones de comercio exterior en una única plataforma, en la cual participasen las distintas entidades que tienen lugar proceso, como exportadores, importadores, agencias de aduana, Servicio Agrícola y Ganadero (SAG), Servicio Nacional de Pesca (SERNAPESCA) y Servicio de Salud, por mencionar sólo algunas. Esto quiere decir que, ante la necesidad que presentan los actores de realizar operaciones de exportación o importación, y que requieran la certificación y o el visto bueno de alguna de esas entidades, podría recurrir a una ventanilla única para conseguirlos, reduciendo así los tiempos que toma el ingreso repetido de información. De hecho, uno de los aspectos que ha influido en los esfuerzos por implementar una VUCE tiene que ver con el desempeño del país agilizando los trámites de comercio exterior en relación a su apertura comercial (pues Chile es el país que cuenta con más acuerdos de libre comercio suscritos en el mundo): para 2014, por ejemplo, un proceso de exportación usual desde Chile requería, en promedio, la tramitación de cinco documentos y tomaba 15 días, y la importación requería igual cantidad de documentos, pero 12 días; en cambio, en un país con menor grado de apertura comercial, como República Dominicana, se requería normalmente la gestión de 4 
documentos para la exportación y 5 para la importación, tomando 8 días el primer procedimiento y 10 días el segundo (Banco Mundial, 2014). Por ende, el nivel de apertura comercial chileno no parece condecirse con la facilitación que ofrece al comercio. Así, pues, se constituyó la VUCE llamada Sistema Integrado de Comercio Exterior (SICEX) en el año 2011. Éste, sin embargo, aún no es del todo implementado por los distintos actores ("Facilitación de Comercio", 2018).

\section{DISCUSIÓN FINAL}

El vínculo entre cambio tecnológico y facilitación del comercio experimentado en otros contextos nacionales -principalmente, en vías de desarrollo-, ha sido analizado, principalmente, poniendo en relieve una relación causal y lineal: las TIC contribuirían a facilitar el comercio, mediante la agilización de los procedimientos aduaneros. Los trabajos que adhieren a esa línea ahondan en los beneficios que esa relación ha producido en las pequeñas y medianas empresas que realizan operaciones de comercio exterior. Así ocurre con los trabajos de Yang (2010), sobre Corea del Sur; Wijayasiri y Jayaratne (2010), en el caso de Sri Lanka; y Rana (2012) sobre India, por mencionar algunos. En cuanto a África, el tenor es similar: Makunike (2015), por ejemplo, observa que las TIC implementadas en aduanas del sur y del oriente africano han generado beneficios para diversos actores del despacho aduanero. Para el caso de América Latina, por otro lado, los trabajos sobre facilitación del comercio no han profundizado en su relación con el cambio tecnológico y se han centrado, sobre todo, en el desempeño de los países (Volpe, 2017).

A diferencia de esos abordajes, que enfrentan el cambio tecnológico desde el punto de vista de su impacto en la facilitación del comercio internacional, este trabajo se orienta por la perspectiva de las transiciones sociotécnicas, exhibiendo un tránsito hacia el establecimiento de un "régimen socio-técnico de facilitación del comercio". Este tránsito muestra que la implementación de tecnologías ocurre en un contexto de evolución experimentada en distintos ámbitos, situando el cambio tecnológico (incorporación de las TIC) como apenas uno de ellos, entre los que se incluyen, por ejemplo: incorporación de nuevos actores, cambio de política comercial y decisiones de organismos multilaterales. Todo ello implica el reconocimiento de una relación multicausal, en la cual las TIC, a la vez, impactan y son impactadas por esos ámbitos. En consecuencia, se da cuenta de un contexto socio-técnico y no tecnológicamente determinista en el ámbito de las operaciones de comercio exterior.

\section{CONCLUSIONES}

A partir de los resultados de la investigación, es posible definir estas conclusiones: 1) el paisaje socio-técnico muestra una trayectoria que influye en el establecimiento del régimen socio-técnico de facilitación del comercio, mediante la institucionalización de organizaciones y normas internacionales que lo promueven; por otro lado, la estrategia de liberalización comercial, germinada en la década de 1970, pero profundizada a partir de la década de 1990, igualmente colabora en la estructuración del régimen; 2 ) es posible advertir, también, la coevolución de aspectos propios de un régimen que se instala, como: participación de distintos actores, implementación de nuevas tecnologías y cambios institucionales, que se han estructurado en torno a la facilitación del comercio fundada en tecnologías de la información; 3) el desarrollo del comercio exterior puede analizarse en su dimensión socio-técnica; particularmente, este tipo de análisis permite distinguir las trayectorias de distintos elementos propios del régimen socio-técnico de facilitación del comercio, cuestión que en la actualidad parece relevante para un país con apertura comercial como Chile, siendo también importante en relación a las formas de adaptación o apropiación de las tecnologías de la información.

\section{AGRADECIMIENTOS}

El autor agradece el apoyo prestado por la Universidad Tecnológica Metropolitana, a través del "Concurso Interno de Fomento a la Investigación y Creación 2014", Proyecto Línea 1: "Innovación tecnológica en el Servicio Nacional de Aduanas de Chile: desde los primeros computadores hasta el SICEX y el programa OEA (1961-2014)".

\section{REFERENCIAS}

Allard, R., Presentación del Director Nacional de Aduanas, Sr. Raúl Allard N., con Ocasión de la Celebración del Día Internacional de Aduanas, Boletín Oficial Servicio Nacional de Aduanas, (133), 1-27 (2004)

Banco Central de Chile., "Base de Datos Estadísticos", acceso el 10 de febrero de 2019 (2019)

Banco Mundial., Doing Business 2015. Going Beyond Efficiency, 12th edition, 167-230, The World Bank, Washington DC, USA (2014)

Creswell, J., Qualitative Inquiry \& Research Design. Choosing Among Five Approaches, 2nd Edition., 147-155, Sage Publications, Thousand Oaks, United States (2007) 
De Sá Porto, P. y C. Morini, Trade Facilitation, the Authotized Economic Operator and the Single Window: A Gravity Model Approach, Customs Scientific Journal, ISSN: 2518-1599, 4(2), 44-53 (2014)

Dennis, A. y B. Shepherd, Trade Facilitation and Export Diversification, doi: 10.1111/j.1467-9701.2010.01303.x, The World Economy, 34(1), 101-122 (2011)

Dirección General de Relaciones Económicas Internacionales, Chile: 20 años de Negociaciones Comerciales, Dirección de Relaciones Económicas internacionales, 29-187, Santiago de Chile, Chile (2009)

El Funcionamiento del Computador Electrónico 1401 es un Gran Paso para la Estadística, Aduana, XXIV(108), 27 (1962)

Facilitación de Comercio, Boletín Informativo Sistema Integrado de Comercio Exterior, julio/agosto 2018, 3, (2018)

Fuentes, H., El Nuevo Sistema Operativo Aduanero, Chile Portuario, 3(6), 81 (1979)

Geels, F.W. y R. Kemp, Dynamics in Socio-technical Systems: Typology of Change Processes and Contrasting Case Studies, doi: 10.1016/j.techsoc.2007.08.009, Technology in Society 29(4), 441-455 (2007)

Geels, F.W., A Socio-technical Analysis of Low-carbon Transitions: Introducing the Multi-level perspective into Transport Studies, doi: 10.1016/j.jtrangeo.2012.01.021, Journal of Transport Geography 24, 471-482 (2012)

Geels, F.W., Co-evolution of Technology and Society: the Transition in Water Supply and Personal Hygiene in the Netherlands (1850-1930) -A Case Study in Multi-level Perspective, doi: 10.1016/j.techsoc.2005.04.008, Technology in Society, 27(3), 363-397 (2005b)

Geels, F.W., Technological Transitions as Evolutionary Reconfiguration Processes: a Multi-level Perspective and a Case Study, doi: 10.1016/S0048-7333(02)00062-8, Research Policy, 31 (8-9), 1257-1274 (2002)

Geels, F.W., The Dynamics of Transitions in Socio-technical Systems: a Multi-level Analysis of the Transition Pathway from Horse-drawn Carriages to Automobiles (1860-1930), doi: 10.1080/09537320500357319, Technology Analysis \& Strategic Management, 17(4), 445-476 (2005a)

Grainger, A., Trade Facilitation: A Conceptual Review, Journal of World Trade, ISSN: 1011-6702, 45(1), 39-62 (2011)

Hernández, R., Fernández, C., Baptista, M., Metodología de la Investigación, 5ta edición, 76-80, McGraw-Hill / Interamericana Editores S.A. de C.V., México DF, México (2010)

Hughes, T., Networks of Power, The John Hopkins University Press, London, England (1983)

Katos, A., The Influence of Information and Communication Technologies on Enabling Trade: A Cross-Country Investigation, Journal of Information Technology Impact, ISSN 1098-139X, 10(1), 15-24 (2010)

Kennedy, R., Queremos, para Chile, lo Mejor, Aduana, XXVI(111), 13 (1964)

Llamazales, J., Algunos Alcances sobre los Nuevos Formularios para la Declaración de Importación y Liquidación-Giro Comprobante de Pago, Boletín Oficial Servicio Nacional de Aduanas, (3), 75-77 (1985)

Makunike, C., The Impact of Information and Communication Technology on Trade Facilitation: A Case Study of East and Southern Africa, Customs Scientific Journal, ISSN 2518-1599, 5(1), 77-88 (2015)

Mewes, E., Charla del Señor Presidente de la Cámara Aduanera, don Eduardo Mewes Ramírez, en el Solemne Acto de Apertura de la Asamblea General Ordinaria de la Institución, el Viernes 28 de noviembre de 1980, Revista de Comercio Exterior, IV(39), 18-23 (1980)

Moïsé, E. y S. Sorescu, Trade Facilitation Indicators: The Potential Impact of Trade on Developing Countries' Trade, doi: 10.1787/5k4bw6kg6ws2-en, OCDE Trade Policy Papers, 144 (2013)

Moïsé, E., The Costs and Challenges of Implementing Trade Facilitation Measures, doi: 10.1787/5k46hzqxt8jh-en, OCDE Trade Policy Papers, 157 (2013)

Moradhasel, N., A. H. Mozayani, S. H. Paryab, Impact of ICT on Business Environment and Trade Facilitation, Quarterly Journal of New Economy \& Commerce, ISSN: 1735-6415, 4(14), 39-63 (2008)

Organización Mundial de Comercio (OMC)., "WTO: A training package; What is Trade Facilitation?". < http://www.wto.org/english/thewto_e/whatis_e/eol/e/wto02/wto2_69.htm\#note2>, 1998, acceso el 2 de agosto de 2006 (1998).

Rana, V.S., An Innovative Use of Information \& Communication Technology (ICT) in Trade Facilitation in India, BVICAM's IJIT, ISSN 0973 - 5658, 4(2), 492-495 (2012)

Tellería, H., Discurso del Presidente de la Cámara Aduanera de Chile Señor Hernán Tellería Ramírez.Acto de Clausura del V Congreso de la Asociación Americana de Profesionales Aduaneros, Chile Portuario, 1(1), $50-52$ (1977)

Valencia, F., C. Marulanda y M. López, Gobierno de las Tecnologías de la Información. Uso y Prácticas en las Entidades Públicas del Triángulo del Café, Colombia, doi: 10.4067/S0718-07642018000300249, Información Tecnológica, 29(3), 249-256 (2018)

Volpe, C., Cómo Salir del Laberinto Fronterizo: Una Evaluación de las Iniciativas de Facilitación del Comercio en América Latina y El Caribe. Informe Especial sobre Integración y Comercio, 1-41, Banco Interamericano de Desarrollo, Washington, Estados Unidos (2017) 
Wijayasiri, J. y S. Jayaratne, The Impact of Information Technology in Trade Facilitation on Small and Medium-Sized Enterprises in Sri Lanka, en The Development Impact of Information Technology in Trade Facilitation, Studies in Trade and Investment, 69, por United Nations ESCAP, United Nations, ISSN: 1020-3516, pp. 177-214, New York, USA (2010)

WT/L/940., Protocolo de enmienda del Acuerdo de Marrakech por el que se establece la Organización Mundial del Comercio, 1-3, Ginebra, Suiza (2014)

Yang, J., Small and Medium-Sized Enterprise Adjustments to Information Technology in Trade Facilitation: The Experience of The Republic of Korea, en The Development Impact of Information Technology in Trade Facilitation, Studies in Trade and Investment, 69, por United Nations ESCAP, United Nations, ISSN: 1020-3516, pp. 69-104, New York, USA (2010)

Zaki, C., How does trade facilitation affect international trade? doi: 10.1057/ejdr.2014.36, European Journal of Development, Research, 27(1), 156-185 (2015) 
